\title{
Distributional Properties of Order Statistics and Record Statistics
}

\author{
*A. H. KHAN AND IMTIYAZ A. SHAH \\ Department of Statistics and Operations Research \\ Aligarh Muslim University, Aligarh 202 002, India \\ *Email: ahamidkhan@ rediffmail.com
}

\begin{abstract}
Distributional properties of the order statistics, upper and lower record statistics have been utilized to characterize distributions of interest. Further, one sided random dilation and contraction are utilized to obtain the distribution of non-adjacent ordered statistics and also their important deductions are discussed.
\end{abstract}

Keywords: Lower and upper record statistics, Order statistics, Random dilation and contraction, Characterization of distributions, Exponential, Weibull, Inverse Weibull, Pareto, Power function and Gumbel distributions.

Mathematics Subject Classification 62E10; 62E15; 62G30.

\section{Introduction}

Let $X_{1}, X_{2}, \cdots$ be a random sample from a continuous population having the probability density function $(p d f) f(x)$ and the distribution function $(d f) F(x)$. Then the $p d f$ and the $d f$ of $X_{L(r)}$, the $r^{t h}$ lower record is given as [Ahsanullah (1995), Arnold et al. (1998)]

and

$$
f_{X_{L(r)}}(x)=\frac{1}{(r-1) !}[H(x)]^{r-1} f(x)
$$

where

$$
F_{X_{L(r)}}(x)=e^{-H(x)} \sum_{j=0}^{r-1} \frac{[H(x)]^{j}}{j !}
$$

$$
H(x)=-\ln F(x)
$$

The $p d f$ of $X_{U(r)}$, the $r^{\text {th }}$ upper record is [Ahsanullah (1995), Arnold et al. (1998)]

and

$$
f_{X_{U(r)}}(x)=\frac{1}{(r-1) !}[R(x)]^{r-1} f(x)
$$

$$
\bar{F}_{X_{U(r)}}(x)=1-F_{X_{U(r)}}(x)=e^{-R(x)} \sum_{j=0}^{r-1} \frac{[R(x)]^{j}}{j !}
$$




\section{A. H. Khan,Imtiyaz A. Shah}

where

$$
R(x)=-\ln \bar{F}(x), \bar{F}(x)=1-F(x)
$$

The $p d f$ and $d f$ of $X_{r: n}$, the $r^{\text {th }}$ order statistic from a sample of size $n$ is given as [Arnold et al. (1992), David and Nagaraja (2003)]

and

$$
f_{X_{r: n}}(x)=\frac{n !}{(r-1) !(n-r) !}[F(x)]^{r-1}[1-F(x)]^{n-r} f(x)
$$

$$
F_{X_{r: n}}(x)=\sum_{j=r}^{n}\left(\begin{array}{c}
n \\
j
\end{array}\right)[F(x)]^{j}[1-F(x)]^{n-j}
$$

If support of the distribution $F(x)$ be over $(\alpha, \beta)$, then by convention, we will write

$$
\begin{array}{ll}
X_{0: n}=\alpha, & X_{n: n-1}=\beta \\
X_{U(0)}=\alpha, & X_{L(0)}=\beta
\end{array}
$$

Alzaid and Ahsanullah (2003) have characterized distributions using distributional properties of adjacent lower records, upper records and order statistics. Castaño-Martínez et al. (2010) have extended the result for non-adjacent case using integral equations rather than differential equations, emphasizing that it is almost impossible to find the solution using differential equations. We, in this paper, have considered the cases for non-adjacent records and order statistics, which Castaño-Martínez et al. (2010) claim that it is still an open problem, with simpler proofs using differential equations and have also considered random dilation and contraction to obtain the distributions of lower records, upper records and order statistics.

We assume that the $d f$ is differentiable w.r.t. its arguments. Further, it is noted that if $Y$ is a measurable function of $X$ with the relation

$$
Y=h(X)
$$

then

(i) $\quad Y_{r: n}=h\left(X_{r: n}\right)$

(ii) $\quad Y_{U(r)}=h\left(X_{U(r)}\right)$

(iii) $Y_{L(r)}=h\left(X_{L(r)}\right)$

If $h$ is an increasing function and

(i) $\quad Y_{n-r+1: n}=h\left(X_{r: n}\right)$

(ii) $\quad Y_{U(r)}=h\left(X_{L(r)}\right)$

(iii) $\quad Y_{L(r)}=h\left(X_{U(r)}\right)$

If $h$ is a decreasing function

Also for simplicity, we shall denote

(i) $\quad X \sim \operatorname{Gum}(\alpha)$

if $X$ has a Gumbel distribution with the $d f$

$$
F(x)=e^{-e^{-\alpha x}}, \quad-\infty<x<\infty, \alpha>0
$$

(ii) $\quad X \sim \operatorname{Wei}(\alpha)$

if $X$ has a Weibull distribution with the $d f$ 


$$
F(x)=1-e^{-x^{\alpha}}, \quad 0<x<\infty, \alpha>0
$$

(iii) $\quad X \sim \operatorname{in} W(\alpha)$

if $X$ has an inverse Weibull distribution with the $d f$

$$
F(x)=e^{-x^{-\alpha}}, \quad 0<x<\infty, \alpha>0
$$

(iv) $X \sim \exp (\alpha)$

if $X$ has an exponential distribution with the $d f$

$$
F(x)=1-e^{-\alpha x}, \quad 0<x<\infty, \alpha>0
$$

(v) $\quad X \sim \operatorname{Par}(\alpha)$

if $X$ has a Pareto distribution with the $d f$

$$
F(x)=1-x^{-\alpha}, \quad 1<x<\infty, \alpha>0
$$

(vi) $\quad X \sim \operatorname{pow}(\alpha)$

if $X$ has a power function distribution with the $d f$

$$
F(x)=x^{\alpha}, \quad 0<x<1, \alpha>0
$$

It may further be noted that

$$
\begin{array}{ll}
\text { if } & \log X \sim \operatorname{Gum}(\alpha) \text { then } X \sim \operatorname{in} W(\alpha) \\
\text { if } & -\log X \sim \operatorname{Gum}(\alpha) \text { then } X \sim \operatorname{Wei}(\alpha) \\
\text { if } & \log X \sim \exp (\alpha) \text { then } X \sim \operatorname{Par}(\alpha) \\
\text { and if } & -\log X \sim \exp (\alpha) \text { then } X \sim \operatorname{pow}(\alpha)
\end{array}
$$

\section{Characterizing results based on lower records}

Theorem 2.1. Let $X_{L(s)}$ be the $s^{\text {th }}$ lower record statistic from a continuous population with the $p d f f(x)$ and the $d f F(x)$, then

$$
X_{L(s-j)} \stackrel{d}{=} X_{L(s)}+Y_{j: s-1}, \quad j=s-r-1, s-r ; 1 \leq r<s
$$

where $Y_{j: s-1}$ is the $j^{\text {th }}$ order statistic from a sample of size $(s-1)$, drawn from $\exp (\alpha)$ distribution and is independent of $X_{L(s)}$ if and only if $X_{1} \sim \operatorname{Gum}(\alpha)$.

Proof. To prove the necessary part, let the moment generating function $(m g f)$ of $X_{L(r)}$ be $M_{X_{L(r)}}(t)$, then

$$
X_{L(r)} \stackrel{d}{=} X_{L(s)}+Y
$$

implies

$$
M_{X_{L(r)}}(t)=M_{X_{L(s)}}(t) \cdot M_{Y}(t)
$$

Since for $\operatorname{Gum}(\alpha)$ distribution.

$$
M_{X_{L(r)}}(t)=\frac{\Gamma\left(r-\frac{t}{\alpha}\right)}{\Gamma(r)}
$$

Therefore, 


$$
M_{Y}(t)=\frac{M_{X_{L(r)}}(t)}{M_{X_{L(s)}}(t)}=\frac{\Gamma(s)}{\Gamma(r)} \frac{\Gamma\left(r-\frac{t}{\alpha}\right)}{\Gamma\left(s-\frac{t}{\alpha}\right)}
$$

But this is the $m g f$ of $Y_{s-r: s-1}$, the $(s-r)^{t h}$ order statistic from a sample of size $(s-1)$ drawn from $\exp (\alpha)$ and the result follows.

For the proof of sufficiency part, note that the $p d f$ of $X_{L(r)}$ by the convolution method is

$$
\begin{aligned}
f_{X_{L(r)}}(x) & =\int_{0}^{x} f_{X_{L(s)}}(u) f_{V_{s-r: s-1}}(x-u) d u \\
& =\frac{\alpha}{B(r, s-r)} \int_{0}^{x} e^{-\alpha r(x-u)}\left[1-e^{-\alpha(x-u)}\right]^{s-r-1} f_{X_{L(s)}}(u) d u
\end{aligned}
$$

Differentiating both the sides w.r.t. $x$, we have for $s>r+1$

$$
\begin{aligned}
\frac{d}{d x} f_{X_{L(r)}}(x) & =\frac{\alpha^{2}}{B(r, s-r)} \int_{0}^{x}\left\{(s-r-1) e^{-\alpha(r+1)(x-u)}\left[1-e^{-\alpha(x-u)}\right]^{s-r-2}\right. \\
& \left.-r e^{-\alpha r(x-u)}\left[1-e^{-\alpha(x-u)}\right]^{s-r-1}\right\} f_{X_{L(s)}}(u) d u \\
& =\alpha r\left[f_{X_{L(r+1)}}(x)-f_{X_{L(r)}}(x)\right]
\end{aligned}
$$

in view of (2.3).

For $s=r+1$,

$$
\begin{aligned}
\frac{d}{d x} f_{X_{L(r)}}(x) & =\frac{\alpha}{B(r, 1)} f_{X_{L(r+1)}}(x)-\alpha r f_{X_{L(r)}}(x) \\
& =\alpha r\left[f_{X_{L(r+1)}}(x)-f_{X_{L(r)}}(x)\right]
\end{aligned}
$$

and therefore, for $s \geq r+1$,

$$
f_{X_{L(r)}}(x)=\alpha r\left[F_{X_{L(r+1)}}(x)-F_{X_{L(r)}}(x)\right]
$$

This in view of (1.1) and (1.2) reduces to,

$$
\frac{1}{\Gamma(r)}[H(x)]^{r-1} f(x)=\frac{\alpha}{\Gamma(r)}[H(x)]^{r} F(x)
$$

and we get,

$$
f(x)=\alpha H(x) F(x)
$$

or, $\quad \frac{d}{d x}[\ln (-\ln F(x))]=-\alpha$

implying that

$$
F(x)=e^{-e^{-\alpha x}}, \quad-\infty<x<\infty, \alpha>0
$$

and hence the proof.

Remark 2.1. Alzaid and Ahsanullah (2003) have shown that

$$
X_{L(r)} \stackrel{d}{=} X_{L(r+1)}+V
$$

where $V \sim \exp (r)$ if and only if $X_{1} \sim \operatorname{Gum}(\alpha)$.

The expression for the $d f$ in Alzaid and Ahsanullah [2003, (1.5)] should have been as given here in (1.2). 
Corollary 2.1. Let $X_{L(s)}$ be the $s^{\text {th }}$ lower record from a continuous population with the $p d f f(x)$ and the $d f F(x)$, then

$$
X_{L(s-j)} \stackrel{d}{=} X_{L(s)} \cdot Y_{j: s-1}, \quad j=s-r-1, s-r ; 1 \leq r<s
$$

where $Y_{j: s-1}$ is the $j^{\text {th }}$ order statistic from a sample of size $(s-1)$ drawn from the $\operatorname{Par}(\alpha)$ distribution and is independent of $X_{L(s)}$ if and only if $X_{1} \sim \operatorname{in} W(\alpha)$.

Proof. Here the product $X_{L(s)} \cdot Y_{j: s-1}$ in (2.7) is called random dilation of $X_{L(s)}$ (Beutner and Kamps, 2008).

To prove the Corollary note that if

then

$$
\log X_{L(r)} \stackrel{d}{=} \log X_{L(s)}+\log Y_{s-r: s-1}
$$

$$
X_{L(r)} \stackrel{d}{=} X_{L(s)} \cdot Y_{s-r: s-1}
$$

and the result follows by an appeal of (1.10), (1.12), (1.22) and (1.24).

Corollary 2.2. Let $X_{U(s)}$ be the $s^{\text {th }}$ upper record from a continuous population with the $p d f f(x)$ and the $d f F(x)$, then

$$
X_{U(s-j)} \stackrel{d}{=} X_{U(s)} \cdot Y_{s-j: s-1}, \quad j=s-r-1, s-r ; 1 \leq r<s
$$

where $Y_{s-j: s-1}$ is the $(s-j)^{\text {th }}$ order statistic from a sample of size $(s-1)$ drawn from $\operatorname{pow}(\alpha)$ distribution and is independent of $X_{U(s)}$ if and only if $X_{1} \sim \operatorname{Wei}(\alpha)$.

Proof. Here the product $X_{U(s)} \cdot Y_{s-j: s-1}$ in (2.8) is called random contraction of $X_{U(s)}$ (Beutner and Kamps, 2008).

To prove the Corollary, we consider

implying

$$
-\log X_{L(r)} \stackrel{d}{=}-\log X_{L(s)}-\log Y_{s-r: s-1}
$$

$$
X_{U(r)} \stackrel{d}{=} X_{U(s)} \cdot Y_{r: s-1}
$$

and the result follows in view of (1.13), (1.14), (1.23) and (1.25).

\section{Characterization results based on upper records}

Theorem 3.1. Let $X_{U(r)}$ be the $r^{\text {th }}$ upper record statistic from a continuous population with the pdf $f(x)$ and the $d f F(x)$, then

$$
X_{U(r+j)} \stackrel{d}{=} X_{U(r)}+X_{U(j)}, \quad j=s-r-1, s-r ; 1 \leq r<s
$$

where $X_{U(j)}$ is independent of $X_{U(r)}$ if and only if $X_{1} \sim \exp (\alpha)$.

Proof. The necessary part can be proved easily using $m g f$.

To prove the sufficiency part, we proceed here as explained in the Theorem 2.1, to get

$$
f_{X_{U(s)}}(y)=\frac{\alpha^{s-r}}{\Gamma(s-r)} \int_{0}^{y}(y-x)^{s-r-1} e^{-\alpha(y-x)} f_{X_{U(r)}}(x) d x
$$

Differentiating both the sides of (3.2) w.r.t.y, we get

$$
\frac{d}{d y} f_{X_{U(s)}}(y)=\alpha\left[f_{X_{U(s-1)}}(y)-f_{X_{U(s)}}(y)\right]
$$




\section{A. H. Khan,Imtiyaz A. Shah}

Thus, for $s \geq r+1$,

$$
f_{X_{U(s)}}(y)=\alpha\left[F_{X_{U(s-1)}}(y)-F_{X_{U(s)}}(y)\right]
$$

and hence the Theorem, in view of (1.3) and (1.4).

Remark 3.1. Alzaid and Ahsanullah (2003, Remark 1) have shown that for two adjacent upper records

$$
X_{U(2)} \stackrel{d}{=} X_{U(1)}+V
$$

where $V \sim \exp (1)$ if and only if $X_{1} \sim \exp (1)$.

Remark 3.2. Oncel et al. (2005) and Ahsanullah (2006) have shown that

$$
X_{U(r+1)} \stackrel{\boldsymbol{d}}{=} X_{U(r)}+V
$$

where $V \sim \exp (1)$ if and only if $X_{1} \sim \exp (1)$.

Remark 3.3. Castaño-Martínez et al. (2010) have shown that

$$
X_{U(s)} \stackrel{d}{=} X_{U(r)}+V
$$

where $V \sim G a(s-r, 1)$ if and only if $X_{1} \sim \exp (1)$.

Corollary 3.1. Let $X_{U(r)}$ be the $r^{\text {th }}$ upper record from a continuous population with the $p d f f(x)$ and the $d f F(x)$, then

$$
X_{U(r+j)} \stackrel{d}{=} X_{U(r)} \cdot X_{U(j)}, \quad j=s-r-1, s-r ; 1 \leq r<s
$$

where $X_{U(j)}$ is independent of $X_{U(r)}$ if and only if $X_{1} \sim \operatorname{Par}(\alpha)$.

Proof. This can be proved by noting that

$$
\log X_{U(s)} \stackrel{d}{=} \log X_{U(r)}+\log X_{U(s-r)}
$$

implies

$$
X_{U(s)} \stackrel{d}{=} X_{U(r)} \cdot X_{U(s-r)}
$$

in view of (1.11) and (1.24).

Corollary 3.2. Let $X_{L(r)}$ be the $r^{\text {th }}$ lower record from a continuous population with the $p d f f(x)$ and the $d f F(x)$, then

$$
X_{L(r+j)} \stackrel{d}{=} X_{L(r)} \cdot X_{L(j)}, \quad j=s-r-1, s-r ; 1 \leq r<s
$$

where $X_{L(j)}$ is independent of $X_{L(r)}$ if and only if $X_{1} \sim \operatorname{pow}(\alpha)$.

Proof. The Corollary can be proved by considering

$$
-\log X_{U(s)} \stackrel{d}{=}-\log X_{U(r)}-\log X_{U(s-r)}
$$

which implies

$$
X_{L(s)} \stackrel{d}{=} X_{L(r)} \cdot X_{L(s-r)}
$$

with an appeal to (1.15) and (1.25). 


\section{Characterizing results based on order statistics}

Theorem 4.1. Let $X_{r: n}$ be the $r^{\text {th }}$ order statistic from a sample of size $n$ drawn from a continuous population with the $p d f f(x)$ and the $d f F(x)$, then

$$
X_{r+j: n} \stackrel{d}{=} X_{r: n}+X_{j: n-r}, \quad j=s-r-1, s-r ; 1 \leq r<s \leq n
$$

where $X_{j: n-r}$ is independent of $X_{r: n}$ if and only if $X_{1} \sim \exp (\alpha)$.

Proof. The proof of the necessary part is easy.

To prove the sufficiency part, we have

$$
f_{X_{s: n}}(y)=\frac{\alpha}{B(n-s+1, s-r)} \int_{o}^{y} e^{-\alpha(y-x)(n-s+1)}\left[1-e^{-\alpha(y-x)}\right]^{s-r-1} f_{X_{r: n}}(x) d x
$$

Differentiating both the sides of (4.2) w.r.t.y, we get for $s \geq r+1$.

$$
\frac{d}{d y} f_{X_{s: n}}(y)=\alpha(n-s+1)\left[f_{X_{s-1: n}}(y)-f_{X_{s: n}}(y)\right]
$$

and

$$
f_{X_{s: n}}(y)=\alpha(n-s+1)\left[F_{X_{s-1: n}}(y)-F_{X_{s: n}}(y)\right]
$$

and thus (David and Nagaraja, 2003)

$$
F(y)=1-e^{-\alpha y}, \quad \alpha, y>0
$$

Remark 4.1. Alzaid and Ahsanullah (2003) have proved that

$$
X_{r: n} \stackrel{d}{=} X_{r-1: n}+V
$$

where $V \sim \exp (n-r+1)$ if and only if $X_{1} \sim \exp (1)$.

Remark 4.2. Castaño-Martínez et al. (2010) have shown that

$$
X_{s: n} \stackrel{d}{=} X_{r: n}+V
$$

where $V \stackrel{d}{=}-\log W$ with $W \sim \operatorname{Be}(n-s+1, s-r)$ if and only if $X_{1} \sim \exp (1)$.

Corollary 4.1. Let $X_{r: n}$ be the $r^{\text {th }}$ order statistic from a sample of size $n$ drawn from continuous population with the $p d f f(x)$ and the $d f F(x)$, then

$$
X_{r+j: n} \stackrel{d}{=} X_{r: n} \cdot X_{j: n-r} \quad, \quad j=s-r-1, s-r ; 1 \leq r<s \leq n
$$

where $X_{j: n-r}$ is independent of $X_{r: n}$ if and only if $X_{1} \sim \operatorname{Par}(\alpha)$.

Proof. To prove consider

$$
\log X_{s: n} \stackrel{d}{=} \log X_{r: n}+\log X_{s-r: n-r}
$$

and the result follows in view of (1.10) and (1.24).

Corollary 4.2. Let $X_{r: n}$ be the $r^{\text {th }}$ order statistic from a sample of size $n$ drawn from a continuous population with the $p d f f(x)$ and the $d f F(x)$, then

$$
X_{s-j: n} \stackrel{d}{=} X_{s: n} \cdot X_{s-j: s-1}, \quad j=s-r-1, s-r ; 1 \leq r<s \leq n
$$

where $X_{s-j: s-1}$ is independent of $X_{s: n}$ if and only if $X_{1} \sim \operatorname{pow}(\alpha)$.

Proof. To prove the Corollary, we note that

$$
-\log X_{s: n} \stackrel{d}{=}-\log X_{r: n}-\log X_{s-r: n-r}
$$




\section{A. H. Khan,Imtiyaz A. Shah}

implies

$$
X_{n-s+1: n} \stackrel{d}{=} X_{n-r+1: n} \cdot X_{n-s+1: n-r}
$$

or,

$$
X_{r: n} \stackrel{d}{=} X_{s: n} \cdot X_{r: s-1}
$$

and the result follows in view of (1.13) and (1.25). This is case of random contraction.

For $j=1(s=r+1)$, Corollary 4.2 was proved by Wesolowski and Ahsanullah (2004).

Theorem 4.2. Let $X_{r: n}$ be the $r^{\text {th }}$ order statistic from a sample of size $n$ drawn from a continuous population with the $p d f f(x)$ and the $d f F(x)$, then

$$
X_{s-j: n-j} \stackrel{d}{=} X_{s-r: n-r}+X_{r-j: n-j}, \quad j=0,1
$$

where $X_{r-j: n-j}$ is independent of $X_{s-r: n-r}$ if and only if $X_{1} \sim \exp (\alpha)$.

Proof. The proof of the necessary part is easy.

To prove the sufficiency part, we have

$$
f_{X_{s: n}}(y)=\frac{\alpha}{B(n-r+1, r)} \int_{0}^{y}\left[e^{-\alpha(y-x)}\right]^{n-r+1}\left[1-e^{-\alpha(y-x)}\right]^{r-1} f_{X_{s-r: n-r}}(x) d x
$$

Therefore,

$$
\begin{aligned}
\frac{d}{d y} f_{X_{S: n}}(y) & =\frac{\alpha(r-1)}{B(n-r+1, r)} \int_{0}^{y} \alpha\left[e^{-\alpha(y-x)}\right]^{n-r+2}\left[1-e^{-\alpha(y-x)}\right]^{r-2} f_{X_{S-r}: n-r}(x) d x \\
& -\frac{\alpha(n-r+1)}{B(n-r+1, r)} \int_{0}^{y} \alpha\left[e^{-\alpha(y-x)}\right]^{n-r+1}\left[1-e^{-\alpha(y-x)}\right]^{r-1} f_{X_{S-r: n-r}}(x) d x
\end{aligned}
$$

Now since,

$$
\begin{aligned}
& f_{X_{r: n}}(x)=\frac{\alpha}{B(n-r+1, r)}\left[e^{-\alpha x}\right]^{n-r+1}\left[1-e^{-\alpha x}\right]^{r-2}\left[1-e^{-\alpha x}\right] \\
& \quad=\frac{\alpha}{B(n-r+1, r)}\left[e^{-\alpha x}\right]^{n-r+1}\left[1-e^{-\alpha x}\right]^{r-2}-\frac{\alpha}{B(n-r+1, r)}\left[e^{-\alpha x}\right]^{n-r+2}\left[1-e^{-\alpha x}\right]^{r-2}
\end{aligned}
$$

implying that

$$
\frac{\alpha}{B(n-r+1, r)}\left[e^{-\alpha x}\right]^{n-r+2}\left[1-e^{-\alpha x}\right]^{r-2}=\frac{n}{r-1} f_{X_{r-1: n-1}}(x)-f_{X_{r: n}}(x)
$$

which leads to

$$
\frac{d}{d y} f_{X_{s: n}}(y)=\alpha n\left[f_{X_{S-1: n-1}}(y)-f_{X_{S: n}}(y)\right]
$$

and

$$
f_{X_{s: n}}(y)=\alpha n\left[F_{X_{s-1: n-1}}(y)-F_{X_{s: n}}(y)\right]
$$

Further, since (David and Nagaraja, 2003)

$$
\left[F_{X_{s-1: n-1}}(y)-F_{X_{s: n}}(y)\right]=\left(\begin{array}{c}
n-1 \\
s-1
\end{array}\right)[F(y)]^{s-1}[1-F(y)]^{n-s+1}
$$

Thus (4.7) reduces to

$$
f(y)=\alpha[1-F(y)]
$$

implying that

$$
\bar{F}(y)=e^{-\alpha y}
$$

and the Theorem is proved. 
Corollary 4.3. Let $X_{r: n}$ be the $r^{\text {th }}$ order statistic from a sample of size $n$ drawn from continuous population with the $p d f f(x)$ and the $d f F(x)$, then

$$
X_{s-j: n-j} \stackrel{d}{=} X_{s-r: n-r} \cdot X_{r-j: n-j}, \quad j=0,1
$$

where $X_{r-j: n-j}$ is independent of $X_{s-r: n-r}$ if and only if $X_{1} \sim \operatorname{Par}(\alpha)$.

Remark 4.3. For $j=0, r=1$, we get

$$
X_{S: n} \stackrel{d}{=} X_{S-1: n-1} \cdot X_{1: n}
$$

Corollary 4.4. Let $X_{r: n}$ be the $r^{\text {th }}$ order statistic from a sample of size $n$ drawn from continuous population with the $p d f f(x)$ and the $d f F(x)$, then

$$
X_{r: n-j} \stackrel{d}{=} X_{r: s-1} \cdot X_{s: n-j}, \quad j=0,1
$$

where $X_{s: n-j}$ is independent of $X_{r: s-1}$ if and only if $X_{1} \sim \operatorname{pow}(\alpha)$.

The Corollary is proved by an appeal of (1.13) and (1.25).

Remark 4.4. For $j=0, s=n$, we get

$$
X_{r: n} \stackrel{d}{=} X_{r: n-1} \cdot X_{n: n}
$$

as given in Wesolowski and Ahsanullah (2004).

\section{References}

1. Ahsanullah, M. (1995). Record Statistics. New York: Nova Science Publishers, Inc. Commack.

2. Ahsanullah, M. (2006). The generalized order statistics from exponential distribution. Pak. J. Statist. 22(2):121-128.

3. Alzaid, A. A. and Ahsanullah, M. (2003). A characterization of the Gumbel distribution based on Record values. Commun. Statist. Theor. Meth. 32: 2101-2108.

4. Arnold, B.C., Balakrishan, N. and Nagaraja, H. N. (1992). A First Course in Order Statistics. New York: Wiley, USA.

5. Arnold, B. C., Balakrishnan, N. and Nagaraja, H. N. (1998). Records. New York: Wiley, USA.

6. Beutner, E. and Kamps, U. (2008). Random contraction and random dilation of generalized order statistics. Commun. Statist. Theor. Meth. 37:2185-2201.

7. Castaño-Martínez, A., López-Blázquez, F. and Salamanca-Miño, B. (2010). Random translations, contractions and dilations of order statistics and records. Statist: : 1-11.

8. David, H. A. and Nagaraja, H. N. (2003). Order Statistics, New York: John Wiley.

9. Oncel S.Y., Ahsanullah, M., Aliev, F.A. and Aygun, F. (2005). Switching record and order statistics via random contraction. Statist. Probab. Lett. 73: 207-217.

10. Wesolowski, J. and Ahsanullah, M. (2004). Switching order statistics through random power contractions. Aust. N.Z.J. Statist. 46(2): 297-303. 